\title{
Second order and Third order NLO studies of L- alanine crystals grown in aqueous solution of hydrofluoric acid (LAHF)
}

\author{
R.Jothi Mani \\ Aditanar College of Arts and \\ science, Tiruchendur, \\ Tamil Nadu, India.
}

\author{
P. Selvarajan \\ Aditanar College of Arts and \\ science, Tiruchendur, \\ Tamil Nadu, India.
}

\author{
H. Alex Devadoss \\ St.John's college of Arts and \\ science,Palayamkottai, \\ Tamil Nadu, India
}

\begin{abstract}
Nonlinear optics is a fascinating field, which plays a vital role in the emerging field of photonics and optoelectronics. A nonlinear optical crystal of L-alanine grown in aqueous solution of hydrofluoric acid is done by slow evaporation method. L-alanine is an NLO material and it has a Second Harmonic Generation (SHG) efficiency of about 0.3 times that of KDP. To alter the various properties of L-alanine, single crystals of L-alanine have been grown in the aqueous solution of hydrofluoric acid. In this work, Lalanine was admixtured with hydrofluoric acid (LAHF) in the molar ratio of 1:1. The grown crystals were colorless and transparent and they were subjected to various studies for characterization.The third-order nonlinearities of LAHF crystal have been investigated by Z-scan method. The values of nonlinear refractive index $\left(\mathrm{n}_{2}\right)$, the nonlinear absorption coefficient $(\beta)$ and third-order nonlinear susceptibility $\left(\chi^{(3)}\right)$ are estimated for the sample
\end{abstract}

Keywords: L-alanine; admixtured crystal; solution growth; NLO;XRD; SHG; THG.

\section{INTRODUCTION}

In recent years, many significant achievements have been made in the field of nonlinear optics because of the development of new nonlinear optical (NLO) crystals of both organic and inorganic type [1-3]. The need for nonlinear optical materials is much more than other materials because of their significant impact on laser technology, optical communication, optical data storage technology etc [4]. Amino acids are a group of organic compounds containing two functional groups such as amino group and carboxylic group. Most of the amino acids and their complexes belong to the family of organic and semiorganic nonlinear optical (NLO) materials that have potential applications in second harmonic generation (SHG), optical storage, optical communication, photonics, electro-optic modulation, optical parametric amplification, optical image processing. Among the amino acids, L-alanine is the simplest acentric crystal and it is a naturally occurring chiral amino acid with a nonreactive hydrophobic methyl group $\left(\mathrm{CH}_{3}\right)$ as a side chain. Lalanine molecule exists as a zwitterion, where the carboxylic group dissociates and the amino group protonates. Efforts have been made on the amino acid mixed organic-inorganic complex crystals, in order to improve the chemical stability, laser damage threshold and nonlinear optical properties. If Lalanine is mixed with different organic and inorganic acids to form novel materials, it is expected to get improved NLO properties [5]. Some complexes of L-alanine have been recently crystallized and various studies have been investigated by many researchers [6-9]. Keeping this in mind, $\mathrm{L}$-alanine is mixed with hydrofluoric acid to form L-alanine hydrogen fluoride crystal by slow evaporation method. But the results showed that the expected crystal is in fact Lalanine crystal with the altered properties in comparison that of L-alanine grown in water. For the first time We report here the investigations on the second order and third order NLO studies of L-alanine crystals grown in aqueous solution of hydrofluoric acid.

\section{SOLUBILITY AND GROWTH}

The salt of L-alanine admixtured with hydrofluoric acid (LAHF) was synthesized by taking L-alanine (99\% purity) and analar grade hydrofluoric acid in the molar ratio of 1:1 in double distilled water. The dissolved solution was heated at $50{ }^{\circ} \mathrm{C}$ for the synthesis of LAHF salt. The purity of the synthesized salt was further increased by repeated recrystallization. The solubility of the synthesized salt was measured by gravimetrical method [10]. Fig.1 shows the solubility curve for LAHF sample and it is observed that the solubility of LAHF sample in water increases with temperature, exhibiting a positive temperature coefficient of solubility. The saturated solution of the re-crystallized salt of LAHF was prepared in accordance with the solubility data and the calculated amounts of the reactants were thoroughly dissolved in double distilled water and stirred well for about 2 $\mathrm{h}$ using a magnetic stirrer to ensure homogeneous temperature and concentration over entire volume of the solution. The solution was filtered and transferred to crystal growth vessels and crystallization was allowed to take place by slow evaporation method. The harvested crystal of LAHF is shown in the figure 2.

\section{SINGLE CRYSTAL XRD ANALYSIS}

The structure of the grown LAHF crystal was analyzed by employing a Bruker-Nonious MACH3/CAD4 single crystal X-ray diffractometer. From the single crystal Xray diffraction data, it is observed that the LAHF crystal belongs to orthorhombic structure with the lattice parameters $\mathrm{a}=5.759(2) \AA, \mathrm{b}=6.042(4) \AA, \mathrm{c}=12.358(3) \AA, \alpha=\beta=\gamma=$ $90^{\circ}$ and $\mathrm{V}=430.01(1) \AA^{3}$. The obtained structural data for the grown crystal of this work are almost coincided with those of L-alanine crystal and therefore the crystal structure of LAHF crystal is not changed [11].The slight changes in lattice 
parameters may be due to incorporation of admixtured material into the lattice of the crystal.

\section{SECOND HARMONIC GENERATION ANALYSIS (SHG)}

The NLO activity in reference to SHG of a sample can be checked using the Kurtz and Perry method [12].

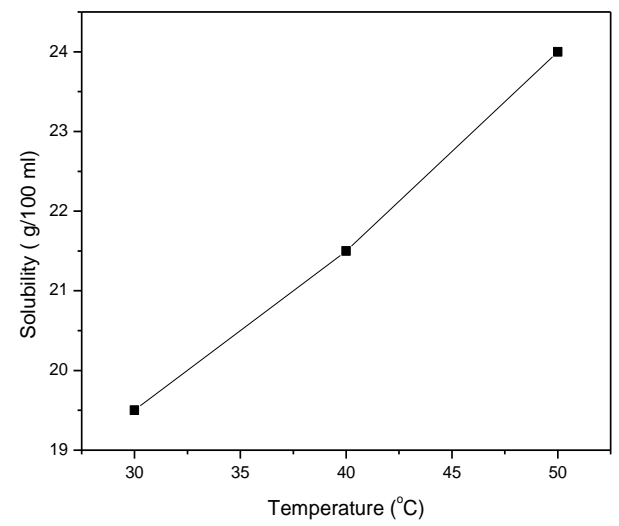

Figure 1. Solubility curve of LAHF sample.

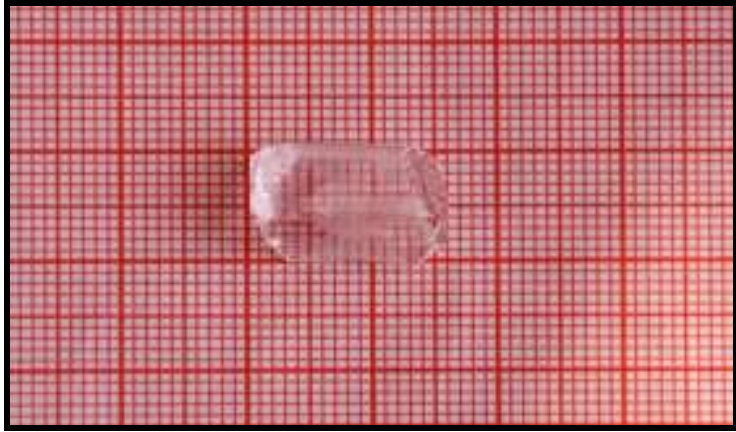

Figure 2. Grown crystal of LAHF.

A high intensity Nd:YAG laser $(\lambda=1064 \mathrm{~nm})$ with a pulse duration of $6 \mathrm{~ns}$ was passed through the powdered sample. The SHG behavior was confirmed from the output of the laser beam having the green emission. $(\lambda=532 \mathrm{~nm})$. The second harmonic generation signal of $9.3 \mathrm{~mJ}$ for LAHF sample was obtained for an input energy of $0.68 \mathrm{~J}$. But the standard KDP crystal gave an SHG signal of $8.8 \mathrm{~mJ}$ for the same input energy. Thus, it is noticed that the SHG efficiency of the grown LAHF sample is 1.05 times that of the standard KDP crystal. It is to be mentioned here that the particle size has been maintained at about 150 microns for both LAHF and KDP samples.

\section{THIRD HARMONIC GENERATION ANALYSIS (THG)}

Third harmonic generation analysis is done by Z-scan technique. It is a standard technique for determining the nonlinear index of refraction $\left(\mathrm{n}_{2}\right)$ and nonlinear absorption coefficient $(\beta)$ of samples $[13,14]$. The study of nonlinear refraction by the Z-scan method depends on the position $(\mathrm{Z})$ of the thin sample under the investigation along a focused Gaussian laser beam. The sample causes an additional focusing and defocusing, depending on whether nonlinear refraction is positive or negative. Measurements of open and closed aperture of the normalized transmittance and sample position $\mathrm{Z}$ has a peak to valley shapes are shown in Fig. 3 and. The curves are characterized by a prefocal transmittance maximum (peak) followed by a postfocal transmittance minimum (valley) intensity. The transmission difference between peak and valley $\left(\Delta \mathrm{T}_{\mathrm{p}-\mathrm{v}}\right)$ is written in terms of phase shift .

$$
\Delta T_{\mathrm{p}-\mathrm{v}=0.406(1-\mathrm{s})} 0.25 \llbracket \Delta \phi \rrbracket
$$

Linear transmittance aperture (S) is calculated using the relation

$$
S=1-\exp \left(\frac{-2 r_{2}^{2}}{w_{e}^{2}}\right)
$$

Where $r_{a}$ is the radius of the aperture and $\omega_{a}$ is the beam radius at the aperture. The third-order nonlinear refractive index $\left(\mathrm{n}_{2}\right)$ of the crystal was calculated by following the relation.

$$
\mathrm{n}_{2}=\frac{\Delta \phi}{\mathrm{KI}_{0} \mathrm{~L}_{\mathrm{sfF}}}
$$

Where $I_{0}$ is the intensity of the laser beam at the focus $(Z=0)$ and $\mathrm{K}=2 \pi / \lambda \quad(\lambda$ is the wavelength of laser beam $)$.

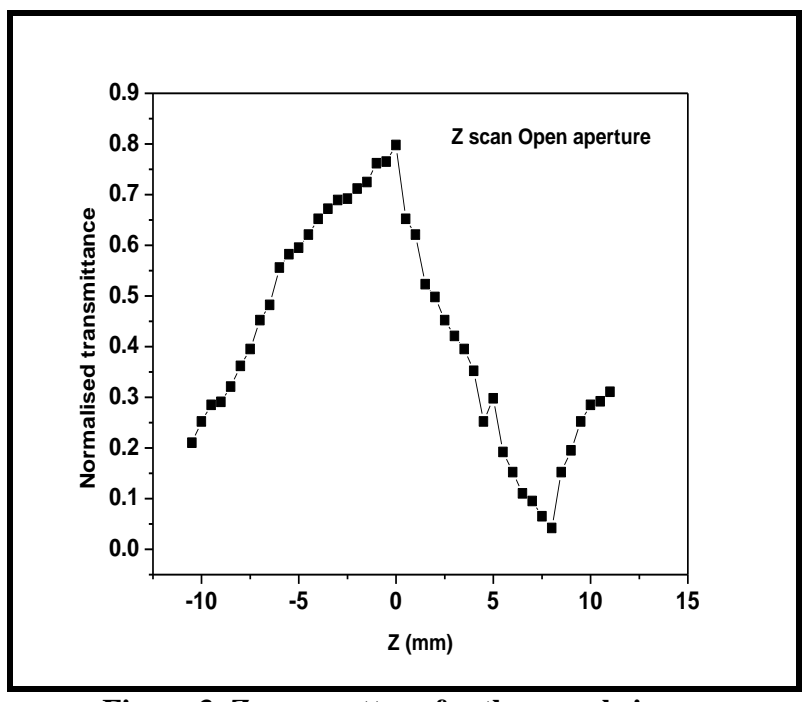

Figure 3. Z-scan pattern for the sample in open aperture

The effective thickness can be calculated using the relation

$$
L_{\text {eff }}=1-\exp (\alpha L / \alpha)
$$

Where $\alpha$ is the linear absorption coefficient and $\mathrm{L}$ is the thickness of the sample.The nonlinear absorption coefficient 


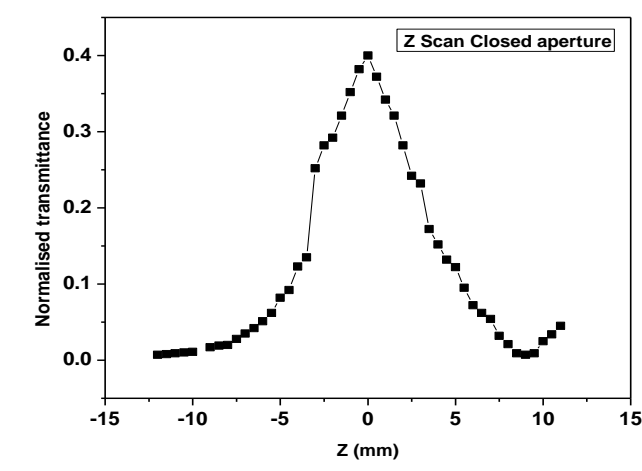

Figure 4. Z-scan patten for the sample in closed aperture

( $\beta$ ) can be calculated using the following relation

$$
\beta=\frac{2 \sqrt{2} \Delta T}{I_{0} L_{\text {eff }}}
$$

Where $\Delta \mathrm{T}$ is the one peak value at the open aperture $\mathrm{Z}$ scan curve. The value of $\beta$ will be negative for saturable absorption and positive for two photon absortion process. The real and imaginary parts of the third order nonlinear optical succeptibility $\left(\chi^{(3)}\right)$ are defined as

$$
\begin{aligned}
& \operatorname{Re} \chi^{(B)}(\text { esu })=\frac{\left.10^{-4}\left[\varepsilon_{0}\left[{ }^{2} n\right\} n^{2}\right]\right)}{\pi}(\mathrm{cm} / W) \\
& \operatorname{Im} \chi^{(B)}(\operatorname{esu})=\frac{10^{-2}\left[\varepsilon_{0}\left[\left[^{2} n\right\} \lambda \beta\right]\right)}{4 \pi^{2}}(\mathrm{~cm} / W) \\
& \mid \chi^{(3)}=\left[(\operatorname{Re} \chi(3))^{2}+(\operatorname{Im} \chi(3))^{2}\right]^{\frac{2}{2}}
\end{aligned}
$$

Where ' $\varepsilon_{0}$ ' is the vacuum permitivity, ' $\mathrm{n}_{0}$ ' is the linear refractive index of the sample and $c$ is the velocity of the light in vacuum. The obtained resutls from the Z-scan measurement for LAHF crystal are

Laser beam wave length $(\lambda)=\mathbf{6 3 2 . 8} \mathbf{n m}$

Spot size diameter in front of the aperture $\left(\omega_{\mathrm{a}}\right)=\mathbf{1} \mathbf{~ c m}$

Aperture radius $\left(\mathbf{r}_{\mathrm{a}}\right)=\mathbf{4} \mathbf{~} \mathbf{m m}$

Incident intensity at the focus $(Z=\mathbf{0})=\mathbf{2} \mathbf{M W} / \mathbf{c m}^{2}$

Effective thickness $\left(\mathrm{L}_{\text {eff }}\right)=\mathbf{0 . 9 8 7} \mathbf{~ m m}$

Nonlinear refractive index $\left(\mathbf{n}_{2}\right)=\mathbf{1 . 0 2 7} \times \mathbf{1 0}^{-12} \mathbf{c ~ m}^{2} / \mathrm{W}$

Nonlinear absorption coefficient $(\boldsymbol{\beta})=\mathbf{0 . 1 0 8} \times 10^{-4} \mathbf{c ~ m} / \mathrm{W}$

Real part of the third-order

nonlinear susceptibility $\operatorname{Re}\left(\chi^{(3)}\right)=0.85 \times 10^{-10}$ esu

Imaginary part of the third- order

nonlinear susceptibility $\operatorname{Im}\left(\chi^{(3)}\right)=4.57 \times \mathbf{1 0}^{-7} \mathrm{esu}$

The third-order nonlinear susceptibility $\left(\chi^{(3)}\right)=6.67 \times 10^{-4}$ esu

The calculated values of the third order nonlinear refractive index $\left(\mathrm{n}_{2}\right)$ is $1.027 \times 10^{-12} \mathrm{c} \mathrm{m}^{2} / \mathrm{W}$ and the positive value of nonlinear refraction reveals the self-focusing nature and nonlinear absorption co-efficient $(\beta)$ is $0.108 \times 10^{-4} \mathrm{c} \mathrm{m} / \mathrm{W}$ and it exhibts the two-photon absorption process. The absolute value of $\left(\chi^{(3)}\right)$ is $6.67 \times 10^{-4}$ esu and this is due to the $\pi$-electron cloud movement from the donor to the acceptor of the L-alanine molecule.

\section{CONCLUSIONS}

Single crystals of LAHF have been grown by slow evaporation solution growth technique and the grown crystals were transparent with a well defined external appearance. The solubility of LAHF crystal is observed to be increasing with increase in temperature. The unit cell parameters for LAHF crystal have been evaluated by single crystal XRD and the structure is confirmed to be orthorhombic. The NLO efficiency of LAHF sample is found to be 1.05 times that of KDP. The values of the third order NLO parameters for the grown sample were determined by Z-scan technique.

\section{ACKNOWLEDGEMENT}

The authors are grateful to Department of Science and Technology (DST), Government of India for the financial support to carry out this work. Also the authors are thankful to the management of Aditanar College of Arts and Science, Tiruchendur for the encouragement given to us to carry out the research work. Research support given by STIC Cochin, Cresent engineering college Chennai and NIT Trichy are gratefully acknowledged.

\section{REFERENCE}

[1] Prasad P.N., Williams D.J., Introduction to Nonlinear Optical effects in Organic Molecules and Polymers, John Wiley \& Sons Inc., New York, USA (1991).

[2] Krishnan C., Selvarajan P.,.Freeda T.H, Mahadevan C.K., Physica B 404 (2009) 289-294.

[3] Jothi Mani R. and selvarajan P., Indian journal of Applied Research Volume: 3 | Issue: 11 | Nov 2013 | ISSN - 2249-555X (446).

[4] Chemla D.S., Zyss J. (Eds.), Nonlinear Optical Properties of Organic Molecules and Crystals, Academic Press, New York (1987).

[5] Lucia Rose A.S.J., Selvarajan P., Perumal S., Materials Chemistry and Physics 130 (2011) 950955

[6] Aravindan A., Srinivasan P., Vijayan N., Gopalakrishnan R., Ramasamy P. Spectrochimica Acta Part A 71 (2008) 297-304.

[7] Rajan Babu D., Jayaraman D., Mohan Kumar R., Jayavel R., J. Cryst. Growth 245 (2002) 121-125.

[8] Razzetti C., Ardoino M., Zanotti L., Zha M., Parorici C., Cryst. Res. Technol. 37 (2002) 456-465.

[9] Justin Raj C., Jerome Das S., J.Crystal Growth 304 (2007) 191-195.

[10] A.S.I.Joy Sinthiya, P.Selvarajan, Ind. J. Appl. Res. 3 (2013) 521-523.

[11] Hema Durga K.K., Selvarajan P., Shanthi D., Int. J. Curr. Res. Rev. 4 (2012) 1168-77

[12] Kurtz S. K., Perry T.T., J. Appl. Phys 39 (1968) 3798-3813.

[13] Sheik-Bahae M., Said A. A., and Van Stryland E. W., Opt. Let. 14 (1989) 955-957.

[14] Kanagasekaran T., Mythili P., Srinivasan P., Nooraldeen A. Y., Palanisamy P., Gopalakrishanan K., Cry. Grow. Des. 8 (2008) 2335-2339 